\title{
Review: over $25 \%$ of people with schizophrenia, psychoses, or severe mental disorders fail to adhere to treatment programmes
}

Nose M, Barbui C, Gray R, et al. Clinical interventions for treatment non-adherence in psychosis: meta-analysis. Br J Psychiatry 2003; 183:197-206.

\section{What proportion of people with psychosis fail to adhere to treatment programmes?}

\section{METHODS}

$\square$

Design: Systematic review with meta-analysis.

Data sources: MEDLINE and PsycINFO from January 1980; 里

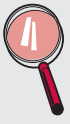

Study selection and analysis: Inclusion criteria: people with schizophrenia, psychoses, or severe mental disorders; participant recruitment in a psychiatric setting and treatment adherence as a primary outcome measure. Exclusions: studies set in general medical wards and emergency rooms, studies on compulsory treatment, and studies examining adherence to initial psychiatric appointments.

娄业

Outcomes: Proportion of people failing to adhere to treatment programmes (either medication or scheduled appointments).

\section{MAIN RESULTS}

Data were extracted from 86 studies (23 796 people). The overall weighted mean rate of non-adherence was $25.78 \%$ (95\% CI 22.46 to 29.10). Patients most associated with non-adherence were young males, with a history of substance abuse, low social functioning, unemployment, or with limited insight into their illness.

\section{CONCLUSIONS}

Over $25 \%$ of people with schizophrenia, psychoses, or severe mental disorders failed to adhere to treatment programmes.

\section{NOTES}

Studies were not assessed for quality. No statistical tests for study heterogeneity were performed and there was substantial variation across studies in patient characteristics and diagnoses; definition of adherence employed; study setting (inpatient, outpatient, or dis-

For correspondence: Dr C Barbui, Department of Medicine and Public Health, Section of Psychiatry, University of Verona, Ospedale Policlinico, Verona, Italy

Sources of funding: not specified. charged patients); study design, and length of follow up. The majority of studies were performed in the United States which may limit the generalisability of results.

\section{Commentary}

his carefully performed meta-analysis focuses on a clinically essential issue in the treatment of schizophrenia and other psychotic disorders. Despite the rapid development of antipsychotic medications in the last decade, it is as important as ever to assess the value of psychosocial interventions tailored for psychoses. In addition, after deinstitutionalisation in psychiatry, adherence to both medication and regular appointments has become crucial for positive outcome during maintenance treatment. The implementation of integrative psychoeducational models has given to both patients and their families a more active role and greater responsibility in the treatment process.

The results of the meta-analysis present both practical and research evidence ${ }^{\prime}$ of the advantages of psychoeducational and other focused interventions on treatment compliance in psychoses. The analysis includes studies comparing standard treatment with interventions aimed at ensuring adherence. All five types of interventions had a favourable effect on patient compliance, most of the studies being psychoeducational programmes. The positive effect was most evident during the first six months of treatment.

The present result can be generalised in patients with schizophrenia and other psychoses. The earlier review by Zygmunt et al ${ }^{2}$ concluded that psychoeducation alone has no effect on patient compliance in schizophrenia, but in their study the control groups could receive other types of interventions as well as standard care. The present findings indicate that there is a further need for education and implementation with structured treatment models that improve continuity in the outpatient care of psychotic disorders. The interventions should be continued long enough to maintain the favourable effect.

Olli Kampman, MD PhD University of Tampere, Medical School, and Seinäjoki Hospital District, Department of Psychiatry, Tampere, Finland

1 Pekkala E, Merinder L. Psychoeducation in schizophrenia. Cochrane Database Syst Rev 2003;lssue 3

2 Zygmunt A, Olfson M, Boyer CA, et al. Interventions to improve medication adherence in schizophrenia. Am J Psychiatry 2002;159:1653-64. 